\title{
Internal multiple attenuation for OBN datasets
}

Roberto Pereira, Mena Ramzy, Petre Griscenco, Benjamin Huard, Luis Cypriano and Adel Khalil, CGG

Copyright 2019, SBGf - Sociedade Brasileira de Geofísica

This paper was prepared for presentation during the $16^{\text {th }}$ International Congress of the Brazilian Geophysical Society held in Rio de Janeiro, Brazil, 19-22 August 2019.

Contents of this paper were reviewed by the Technical Committee of the $16^{\text {th }}$ International Congress of the Brazilian Geophysical Society and do not necessarily represent any position of the SBGf, its officers or members. Electronic reproduction or storage of any part of this paper for commercial purposes without the written consent of the Brazilian Geophysical Society is prohibited.

\section{Abstract}

Ocean bottom node (OBN) technology has emerged as a promising alternative to conventional towed-streamer acquisition. It can provide the full-azimuth long-offset illumination needed for imaging of targets below complex overburdens (Bunting \& Moses, 2016). Areas of such complexity are often affected by the presence of internal multiples. These multiples have detrimental effects on interpretation and amplitude analysis, as observed in examples from the pre-salt regime of the Santos basin, offshore Brazil (Hembd et al., 2011; Cypriano et al., 2015).

A method for predicting internal multiples in streamer acquisitions has been recently introduced (van der Neut \& Wapenaar, 2016; Pereira et al., 2018) and applied to various field datasets (Krueger et al., 2018). In this abstract we extend this method to the case of ocean bottom recordings and apply it to a dataset from the Santos basin.

\section{Introduction}

A typical seismic section from the Santos basin is depicted in Figure 1. Several strong reflectors can be identified in this section: water bottom, post-salt reflectors, top of salt (TOS), stratified salt and base of salt (BOS). These are all potential generators of internal multiples. These multiples can create artifacts in the image of the pre-salt targets, affecting interpretation and contaminating amplitude analysis. The presence of stratified salt in this basin makes it particularly challenging to model internal multiples, since identification of generators is not practical.

In the case of streamer acquisitions, a method for modeling internal multiples has been recently proposed (van der Neut \& Wapenaar, 2016) and applied to datasets from the Santos basin (Pereira et al., 2018; Krueger et al., 2018). It relies on a separation of the data between target and overburden regions. It does not require the identification of particular generators as in the method proposed by Jakubowicz (1998), which has been the standard in the industry to date.

The method proposed can be derived from Marchenko equations defined at the acquisition surface, rather than in the subsurface as in more traditional Marchenko methods for imaging and redatuming (Wapenaar et al., 2014; van der Neut et al., 2015a; Staring et al., 2017).

OBN acquisitions offer many advantages over conventional streamer acquisitions in particular for the imaging of deep targets, including: reduced noise level, broadband signal, long offsets and wide azimuth illumination.

In the following section we revise the derivation of the internal multiple prediction method for the streamer case and generalize it to the case of ocean bottom recordings. We then describe the application to an OBN dataset from the Santos basin showing strong contamination from internal multiples.

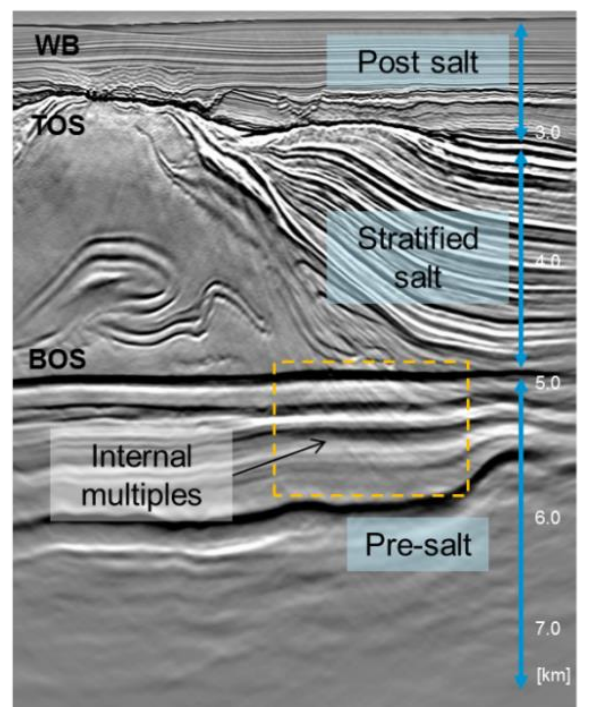

Figure 1 - Migrated seismic section from the Santos basin. Area contaminated by internal multiples in the presalt is highlighted by the dashed box. Internal multiples are generated in the overburden, in particular by the series of reflectors forming the stratified salt.

\section{Method}

Following van der Neut et al. (2015a), we start with the first order correction term for the up-going wavefield defined at a virtual location $x_{h}$ at the subsurface:

$$
G^{-(1)}\left(x_{b}, x_{h} ; t\right)=\left\{\Theta_{t_{d}^{\epsilon}}^{\infty} \mathbf{R}\left(\Theta_{-t_{d}^{\epsilon}}^{+t_{d}^{\epsilon}} \mathbf{R}^{*} \Theta_{-t_{d}^{\epsilon}}^{+t_{d}^{\epsilon}} \mathbf{R}\right) f_{d}\right\}\left(x_{b}, x_{h} ; t\right),
$$

where $t_{d}^{\epsilon}$ is the direct arrival time between $x_{h}$ and $x_{b}$ in the acquisition surface shifted by a small number $\epsilon$ to take into account the size of the wavelet (that is $t_{d}^{\epsilon}=t_{d}-\epsilon$ ). $\Theta_{t_{a}}^{t_{b}}$ is a mute function defined to be equal to 1 between $t_{a}$ and $t_{b}$, and 0 otherwise. $\mathbf{R}$ and $\mathbf{R}^{*}$ are multi-dimensional 
convolution and cross-correlation operators respectively, constructed out of reflection data recorded at the acquisition surface. $f_{d}\left(x, x_{h} ; t\right)$ represents the initial estimate of the focusing function between $x_{h}$ in the subsurface and $x$ at the acquisition surface. It is defined to be the inverse of the direct arrival between those two points:

$$
\delta\left(x_{a}, x_{b}\right)=\int_{\Lambda_{h}} \mathrm{~d} x_{h} G_{d}\left(x_{a}, x_{h} ; t\right) * f_{d}\left(x_{b}, x_{h} ; t\right),
$$

where $x_{a}$ and $x_{b}$ are two given points on the acquisition surface and $\Lambda_{h}$ is a surface separating the data in target and overburden regions.

The expression in equation (1) computes corrections to the up-going wavefield used to construct the image at the location $x_{h}$ in the subsurface. The corrections correspond to artifacts from incorrectly back propagating internal multiples in the data. In principle one would need an infinite series of such terms to completely remove the artifacts. Higher order terms are difficult to compute and more sensitive to data sampling (Staring et al. 2017). Instead, it is preferred to treat eq. (1) as a model and compute filters to adaptively remove artifacts from the initial wavefield (van der Neut et al., 2015b).

We follow the same strategy to construct models for wavefields defined directly at the acquisition surface. Convolving eq. (1) with $G_{d}\left(x_{a}, x_{h} ; t\right)$ and integrating over $x_{h}$ we obtain an expression for a model defined at the surface:

$$
\begin{aligned}
& U_{h}^{(1)}\left(x_{b}, x_{a} ; t\right)=-\int_{\Lambda_{h}} \mathrm{~d} x_{h} G^{-(1)}\left(x_{b}, x_{h} ; t\right) * G_{d}\left(x_{a}, x_{h} ; t\right)= \\
& -\int_{\Lambda_{h}} \mathrm{~d} x_{h}\left\{\Theta_{t_{d}^{\epsilon}}^{\infty} \mathbf{R}\left(\Theta_{-t_{d}^{\epsilon}}^{+t_{d}^{\epsilon}} \mathbf{R}^{*} \Theta_{-t_{d}^{\epsilon}}^{+t_{d}^{\epsilon}} \mathbf{R}\right) f_{d}\right\}\left(x_{b}, x_{h} ; t\right) * G_{d}\left(x_{a}, x_{h} ; t\right) .
\end{aligned}
$$

Looking at the expression above one would be tempted to move $G_{d}$ into the bracket and use eq. (2) to collapse $f_{d}$ to a delta function, which is prevented by the presence of the mute functions. Instead, van der Neut \& Wapenaar (2016) proceed to first project all wavefields to the surface, defining new Marchenko equations at the acquisition level. By properly choosing traveltime functions $t_{0}$ and $t_{h}$ one arrives at the following internal multiple model:

$$
\begin{aligned}
M_{h}\left(x_{b}, x_{a} ; t\right) & =-\left\{\Theta_{t_{h}}^{\infty} \mathbf{R}\left(\Theta_{t_{0}}^{t_{h}} \mathbf{R}^{*} \Theta_{t_{0}}^{t_{h}} \mathbf{R}\right) \delta\right\}\left(x_{b}, x_{a} ; t\right) \\
& =-\left\{\Theta_{t_{h}}^{\infty} \mathbf{R} \Theta_{t_{0}}^{t_{h}} \mathbf{R}^{*} \Theta_{t_{0}}^{t_{h}} R\right\}\left(x_{b}, x_{a} ; t\right) .
\end{aligned}
$$

Intuitively, convolving with $G_{d}$ adds a time corresponding to $t_{d}$. At zero offset, $t_{0}=-t_{d}^{\epsilon}+t_{d}=\epsilon$ and $t_{h}=t_{d}^{\epsilon}+t_{d} \sim$ $2 t_{d}$. Comparing equations (3) and (4) we see that we have effectively collapsed the integral over $x_{h}$ at the expense of redefining the mute functions.

Taking $x_{a}$ to be the shot position and $x_{b}$ to be the receiver position then $R\left(x, x_{a}\right)$ represents a shot record acquired at the surface. The shot-based implementation in the streamer case follows as described in Pereira et al. (2018). The interesting step corresponds to the second mute, after applying the cross-correlation operator $\mathbf{R}^{*}$. At this point we have a virtual shot as defined by lkelle (2006), see Figure 2a. The objective of the mute defined by $t_{0}$ is to separate the primary energy close to zero from the multiple energy appearing at non-zero lag. Figure $2 c$ illustrates a virtual event that should be muted with $t_{0}$ in order to prevent primary leakage in the internal multiple model.
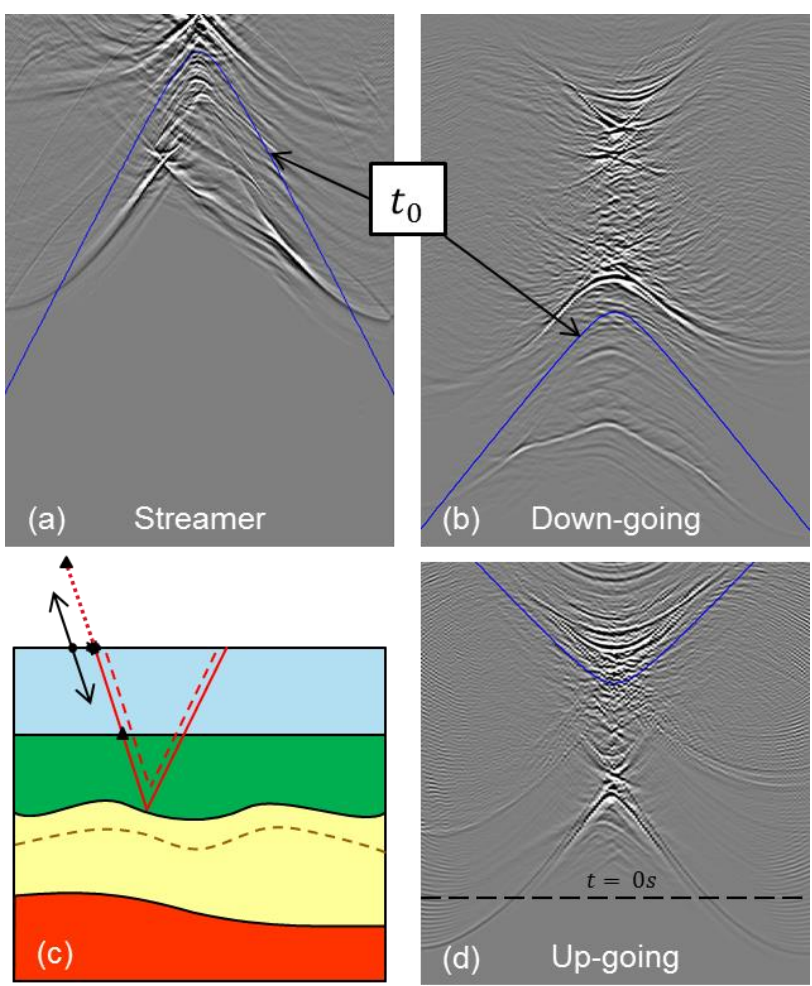

Figure 2 - Examples of mutes in the virtual domain: (a) streamer; (b) and (d) OBN down-going and up-going, respectively. In (c), a virtual event that should be muted with $t_{0}$ is represented. Convolving the last trace with this virtual event would lead to a primary in the internal multiple model.

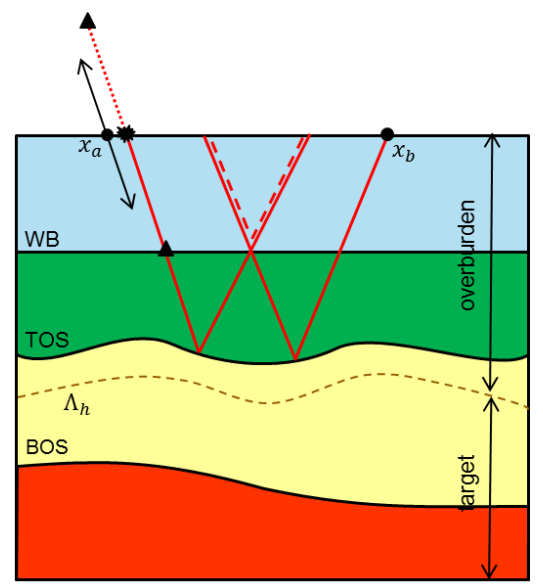

Figure 3 - Cartoon representing equation (4). Possible positions of $x_{a}$ corresponding to the streamer (at surface) and $O B N$ down-going and up-going components (triangles) are represented in the picture. The dashed red line represents a trace coming from $\boldsymbol{R}^{*}$ and the solid line ending at $x_{b}$ represents a trace coming from $\boldsymbol{R}$. The surface $\Lambda_{h}$ that defines target/overburden regions is indicated by the dashed brown line. 
Muting in this virtual domain is precisely what makes it possible to predict internal multiples without identifying generators. Note that for short-period multiples we would need to make $t_{0}$ closer to zero and in turn risk primary leakage in the internal multiple prediction.

Alternatively, in the case of ocean bottom acquisitions, we can take $x_{a}$ to lie on the ocean bottom at a receiver position for the up-going wavefield or at the mirror position for the down-going wavefield (see Figure 3), and $x_{b}$ to be a shot position. The equation is exactly the same where now $R\left(x, x_{a}\right)$ represents data with a source at the sea level surface and a receiver at the ocean bottom. Implementation is now receiver based. The traveltime mutes $t_{0}$ and $t_{h}$ need to be redefined accordingly (see Figure $2 b-d$ ). Note that in this case the operators $\mathbf{R}$ and $\mathbf{R}^{*}$ are still constructed out of reflection data defined at the surface, such as an auxiliary streamer acquisition overlapping with the area covered by the nodes.

\section{OBN dataset example}

We applied the method described above to an OBN dataset from the Santos basin. The water depth is around $2100 \mathrm{~m}$. A towed streamer dataset also covers the same area. The azimuth between the sail lines from the node acquisition and the towed streamer acquisition is about 60 degrees, see Figure 4.

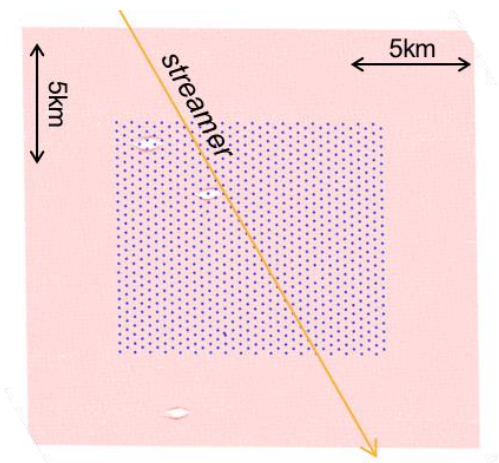

Figure 4 - $O B N$ acquisition. Receiver locations are shown in blue and source carpet in red. Direction of auxiliary streamer acquisition is shown by the orange arrow.

This auxiliary streamer dataset is necessary to construct operators $\mathbf{R}$ and $\mathbf{R}^{*}$ in equation (4). The streamer dataset was pre-processed with a full broadband flow to minimize the spectral difference with respect to the OBN dataset. In particular, designature, source and receiver deghosting and surface related multiple elimination (SRME) were applied to this dataset.

The OBN dataset went through its own broadband preprocessing flow, including PZ sum to separate up- and down-going components, designature, source deghosting and SRME. In the following section, we will show results for the down-going wavefield only.

\section{Internal multiple attenuation}

Aiming to capture all multiples present in this dataset, we decided to produce two internal multiple models, based on different surfaces $\Lambda_{h}$ (Figure 5), one just below the top of salt (TOS) and the second just above the base of salt (BOS). The first model contains the dominant multiples generated by the TOS, while the second model will also have multiples with at least two generators inside the salt.

For simplicity we refer to the two models as the TOS model and BOS model, respectively. An example of an internal multiple predicted by the BOS model but not by the TOS model is shown in red in Figure 5 . We experimented with a surface below the base as well, but in that case, the predicted multiples arrived deeper than the reservoir level.

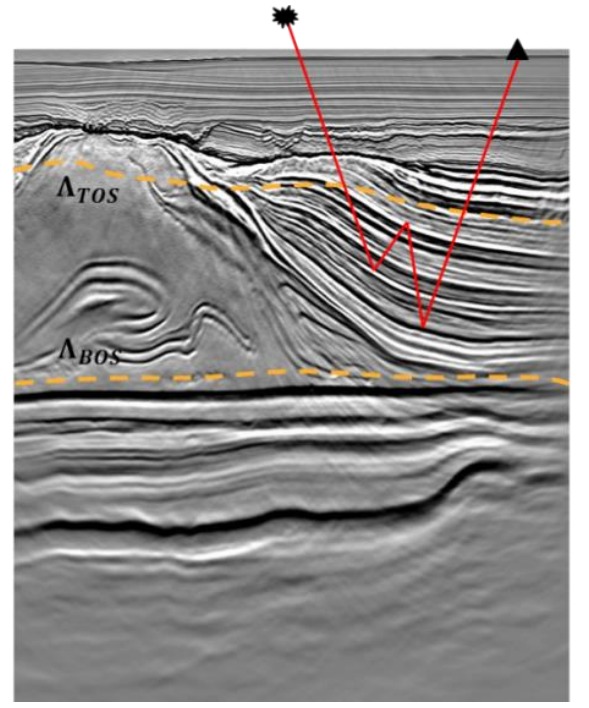

Figure 5 - The two surfaces used to define the internal multiple models. An example of an internal multiple contained in the BOS model but not in the TOS model is shown in red.

Given the different orders of magnitude for multiples present in the BOS model, we decided to construct a third model given by the difference between the BOS and TOS models, referred as BOS-TOS. We then subtract both the multiples from the TOS and BOS-TOS models. The point here is to make the subtraction as orthogonal as possible and allow for different parameterizations in the two passes.

Equation (4) is implemented node by node. The streamer dataset is first interpolated to construct the operators $\mathbf{R}$ and $\mathbf{R}^{*}$. After prediction, we separate input and models in common offset vectors (COVs) and migrate each COV independently.

In order to better discriminate multiple from primary events, subtraction is performed in the migrated COV domain with the help of $3 D$ curvelet filters (Wu \& Hung, 2015). 

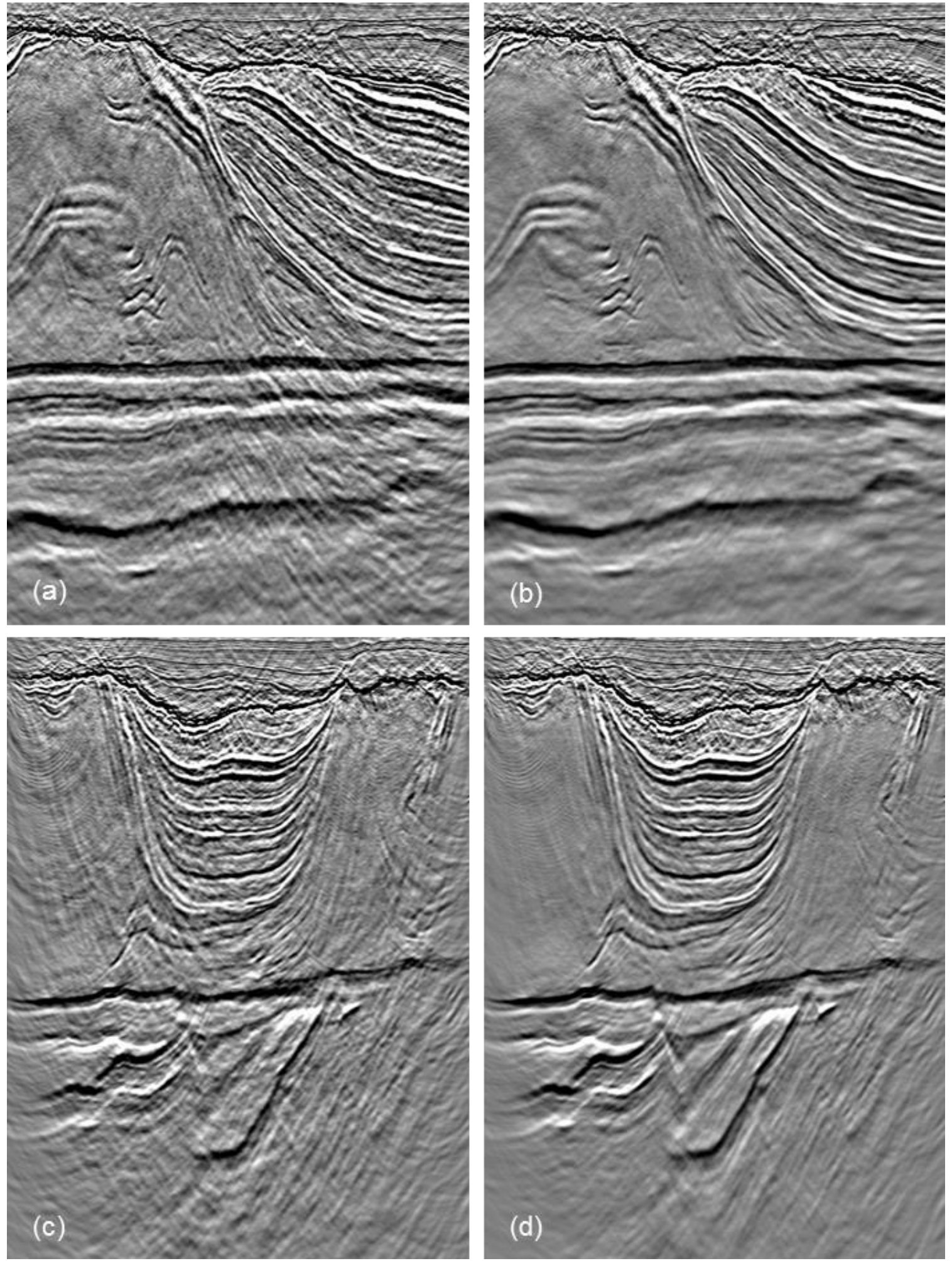

Figure 6 - Internal multiple attenuation results for a near offset COV from the down-going wavefield. (a) and (b): inline section before and after subtraction, respectively. (c) and (d): crossline section before and after subtraction, respectively. 

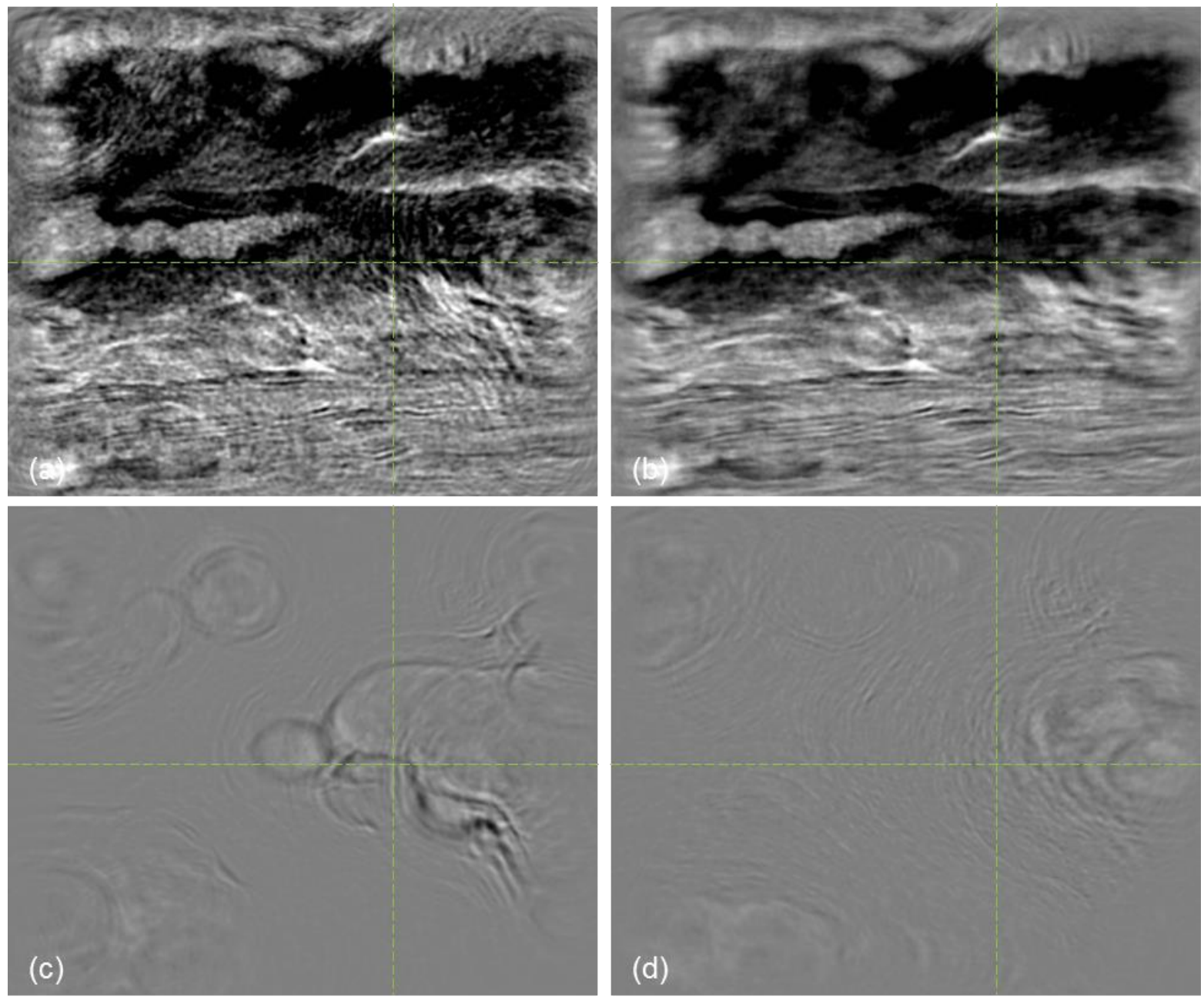

Figure 7 - Depth slices from near offset COV and models. (a) and (b): depth slice before and after subtraction, respectively. (c) and (d): depth slice of the TOS model and the BOS-TOS model, respectively. The dashed green lines indicate positions of inline and crossline sections of Figures 6 and 8.

Figures $6 \mathrm{a}$ and $6 \mathrm{c}$ show an inline and a crossline from a near offset COV. Both images show strong artifacts from internal multiples, in particular in regions below the stratified salt. Figures $6 \mathrm{~b}$ and $6 \mathrm{~d}$ show the result after subtraction. We can observe an improved continuity of pre-salt events and overall a much cleaner image.

Figures $7 \mathrm{a}$ and $7 \mathrm{~b}$ show a depth slice from the same near offset COV before and after subtraction. The depth slice was located just below the BOS. Figure 7 a shows a clear imprint of artifacts from the overburden multiples while in Figure $7 \mathrm{~b}$ most of the artifacts have been removed.

Figures $7 c$ and $7 d$ show the two models used in this subtraction. The TOS model (Figure 7c) contains the dominant multiples while the BOS-TOS model (Figure 7d) shows the artifacts generated by the stratified salt.

Finally, Figures 8a-c show the results of subtraction for an inline section from a near offset stack. A CDP-COV gather with offsets up to $5 \mathrm{~km}$ is shown in Figures $8 \mathrm{~b}-\mathrm{d}$. The mute used to create the near stack includes offsets up to roughly $1.5 \mathrm{~km}$ at the base of salt and opens up to 3 $\mathrm{km}$ at the bottom of the section. We can observe overall a good attenuation of artifacts in this section with some residuals on the right.

\section{Conclusions}

We have generalized a method recently proposed for predicting internal multiples to the case of ocean bottom recordings. Application to an OBN dataset from the Santos basin with strong internal multiples shows a clear improvement in the images.

\section{Acknowledgments}

We would like to thank Petrobras for discussions and technical contributions as well as permission to publish the images. We thank CGG for permission to publish this work. 

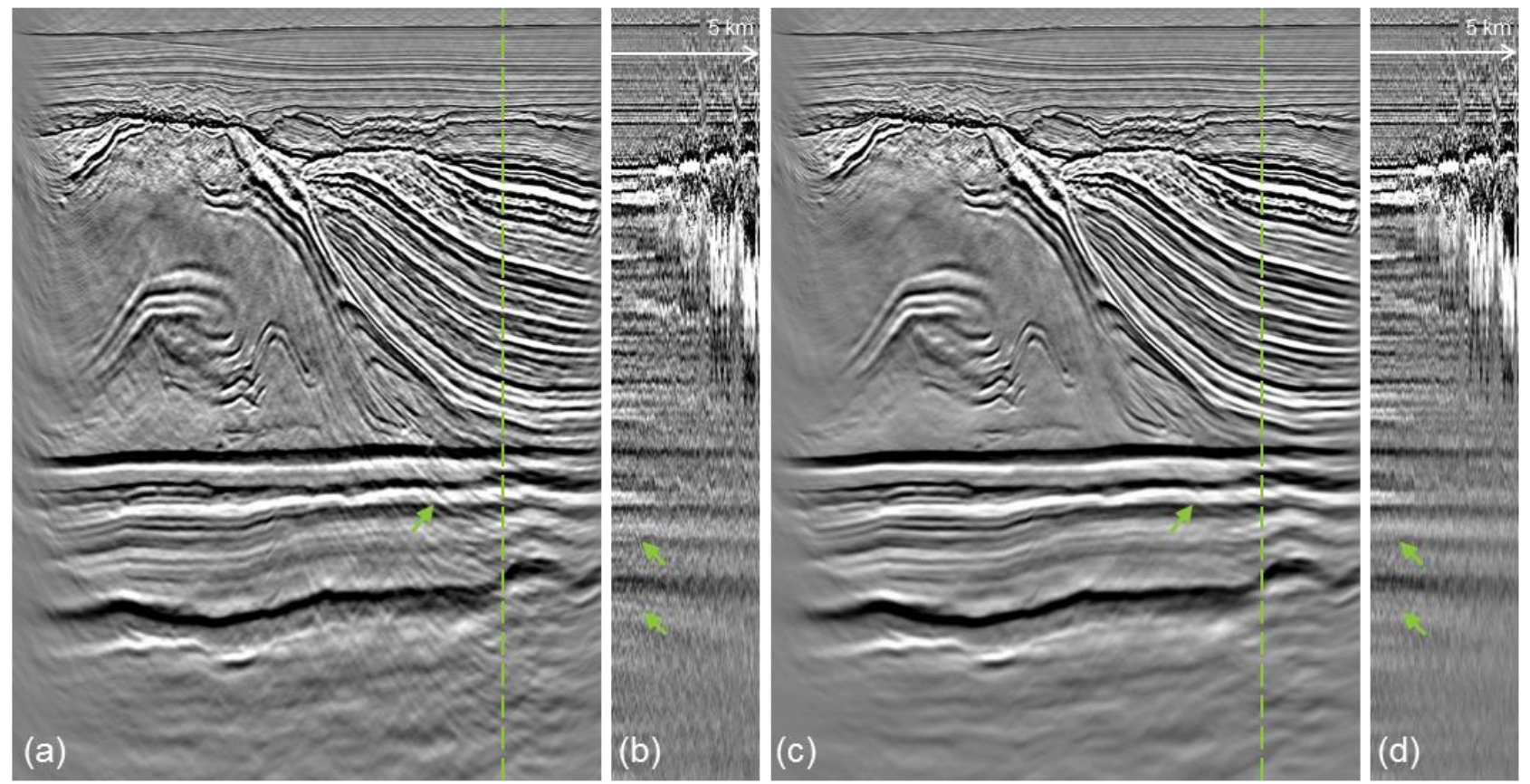

Figure 8 - Inline section from a near offset stack and CDP-COV gathers. (a) and (c): stack before and after subtraction. (b) and (d) gathers before and after subtraction. Gathers are shown up to $5 \mathrm{~km}$ of offset. The position of the gathers is indicated by the dashed green line. Green arrows show internal multiples.

\section{References}

BUNTING, T., \& MOSES, J., 2016, The transformation of seabed seismic. First Break, v34, 59-64.

CYPRIANO, L., MARPEAU, F., BRASIL, R., WELTER, G., PRIGENT, H., DOUMA, H., VELASQUES, M., BOECHAT, J., DE CARVALHO, P., GUERRA, C., \& THEODORO, C., 2015, The impact of inter-bed multiple attenuation on the imaging of pre-salt targets in the Santos basin off-shore Brazil. 77th EAGE Conference \& Exhibition, Extended Abstracts, N114 06.

HEMBD, J., GRIFFITHS, M., TING, C., \& CHAZALNOEL, N., 2011, Application of 3D interbed multiple attenuation in the Santos Basin, Brazil. 73rd EAGE Conference and Exhibition, Extended Abstracts, H047.

IKELLE, L. T., 2006, A construct of internal multiples from surface data only: the concept of virtual seismic events. Geophysical Journal International, 164(2), 383-393.

JAKUBOWICZ, H., 1998, Wave equation prediction and removal of interbed multiples. 68th SEG Annual International Meeting, Expanded Abstracts, 1527-1530.

KRUEGER, J., DONNO, D., PEREIRA, R., MONDINI, D., SOUZA, A., ESPINOZA, J., \& KHALIL, A., 2018, Internal multiple attenuation for four presalt fields in the Santos Basin, Brazil. In SEG Technical Program Expanded Abstracts 2018 (pp. 4523-4527). Society of Exploration Geophysicists.
PEREIRA, R., MONDINI, D., \& DONNO, D., 2018, Efficient 3D internal multiple attenuation in the Santos basin. 80th EAGE Conference and Exhibition, Extended Abstracts, We C 08.

STARING, M., PEREIRA, R., DOUMA, H., VAN DER NEUT, J., \& WAPENAAR, C., 2017, Adaptive doublefocusing method for source-receiver Marchenko redatuming on field data. In SEG Technical Program Expanded Abstracts 2017 (pp. 4808-4812). Society of Exploration Geophysicists.

VAN DER NEUT, J., VASCONCELOS, I., \& WAPENAAR, K., 2015a, On Green's function retrieval by iterative substitution of the coupled Marchenko equations. Geophysical Journal International, 203(2), 792-813.

VAN DER NEUT, J., WAPENAAR, K., THORBECKE, J., SLOB, E., \& VASCONCELOS, I., 2015b, An illustration of adaptive Marchenko imaging. The Leading Edge, 34(7), 818-822.

VAN DER NEUT, J., \& WAPENAAR, K., 2016, Adaptive overburden elimination with the multidimensional Marchenko equation. Geophysics, 81(5), T265-T284.

WAPENAAR, K., THORBECKE, J., VAN DER NEUT, J., BROGGINI, F., SLOB, E., \& SNIEDER, R., 2014, Marchenko imaging. Geophysics, 79(3), WA39-WA57.

WU, X., \& HUNG, B., 2015, High-fidelity adaptive curvelet domain primary-multiple separation. First Break, 33(1), 53-59. 